\title{
A New Analyzing Method for Hyperbolic Telegraph Equation
}

\author{
Imtiaz Ahmad ${ }^{1}$, Ahmed Abouelregal ${ }^{2}$, Hijaz Ahmad ${ }^{3}$, Phatiphat Thounthong ${ }^{4}$, and \\ Mahmoud Abdel-Aty ${ }^{5}$ \\ ${ }^{1}$ University of Swabi \\ ${ }^{2}$ Mansoura University Faculty of Science \\ ${ }^{3}$ NWFP University of Engineering and Technology \\ ${ }^{4}$ King Mongkut's University of Technology North Bangkok \\ ${ }^{5}$ Sohag University Faculty of Science
}

July 19, 2020

\begin{abstract}
We proposed an efficient local differential quadrature method which is based on the radial basis function to the numerical solution of the two-dimensional second-order hyperbolic telegraph equations. The explicit time integration technique is utilized to semi-discretize the model in the time direction whereas the space derivatives of the model is discretized by the proposed local meshless procedure based on multiquadric radial basis function. Numerical experiments on ve test problems are performed with the proposed numerical scheme for rectangular and non-rectangular computational domains. The results obtained show that the proposed scheme solutions are converging extremely faster comparable to different existing protocols.
\end{abstract}

\section{Hosted file}

Manuscript.pdf available at https://authorea.com/users/344043/articles/470720-a-newanalyzing-method-for-hyperbolic-telegraph-equation 